\title{
Do City Size and Population Density Influence Regional Innovation Output Evidence from China?
}

\author{
Cai Shukai, Wang Haochen, and Zhou Xiaohong (iD \\ Department of Economics and Management, Anhui Polytechnic University, Wuhu, Anhui 241000, China \\ Correspondence should be addressed to Zhou Xiaohong; 017050@ahpu.edu.cn
}

Received 30 June 2021; Accepted 19 July 2021; Published 10 August 2021

Academic Editor: Thippa Reddy G

Copyright (c) 2021 Cai Shukai et al. This is an open access article distributed under the Creative Commons Attribution License, which permits unrestricted use, distribution, and reproduction in any medium, provided the original work is properly cited.

\begin{abstract}
This paper proposed a substantial gap to a large-scale population density and city size on regional innovation output. To measure the impact of population density and city size on regional innovation output, this study employs the threshold effect model with panel data of 230 prefectures and cities from 2007 to 2016. Based on the econometric analysis, the results exhibit a positive and significant relationship between population density, city size, and innovation output. This correlation suggests that when one factor increases, the other increases in the parallel direction and vice versa. Moreover, when the city size expands the threshold value of 2.934 percent, the innovation promotes and increases the effects of urban-scale expansion. On the other hand, for medium- and low-density cities, the increase of urban population density has a significant and positive impact on urban innovation output. However, for high-density cities, the increase of population density has no significant impact on innovation output.
\end{abstract}

\section{Introduction}

With the advancement of the new urbanization strategy, the population mobility flows rapidly increase between urban and rural cities, while the population size of Chinese cities is constantly changing. In recent years, the population mobility flows from the first-tier cities such as Beijing, Guangdong, Shanghai, and Tianjin, which increase alongside with the second-tier cities, namely, Nanjing, Chengdu, Suzhou, Dalian, Tianjin, Zhongshan, and Dongguan. However, some uncompetitive urban population continued to flow out and even evolved into "shrinking cities." At the same time, the population density level of different cities also changed accordingly. Due to the unplanned allocation of infrastructure development in many Chinese cities, urbanization is expanding faster than the growing population in these cities, while the population density of Chinese cities shows a downward trend due to the lack of planned development. Nevertheless, the changes in urban population density in different scales are determined on basis of various administrative levels and region-to-region significant disparities [1]. In the urban population density in dominant administrative level, the study examines that the central and eastern regions are growing fast. The city is the core carrier of innovation, and scholars widely believe that innovation is mainly in the city. Moreover, larger cities are the backbone core centers of technological innovation; numerous research scholars extensively acknowledge that innovation is the first place phenomenon in a city. Pred (1996) found the per capita patents of 35 largest cities in the United States from 1860 to 1910 and examined that per capita of the four megacities was approximately four times larger than the national average [2]. According to Duncan et al. (1999), a study of 26 metropolises in the United States investigated that these metropolitan cities not only occupy a large number of territorial areas but also carry a large number of the urban population and generated $75 \%$ of the patents of the national metropolises [3]. Marella et al. (2017) and Wojcik-Popek (2019) explored the links between city size and patents. However, their findings cannot identify external effects $[4,5]$. Oort and Bosma (2012) performed empirical analysis on the data of 14 countries and 110 regions in Europe; they examined the mechanism of the impact of urban scale on innovation, and their findings show that the impact of urban scale on innovation 
is linked by human capital [6]. A study proposed by Carlino et al. (2007) with external factors remaining unchanged found that for every 2 -fold increase in population density in metropolitan cities of the United States, the corresponding patent strength would increase by $20 \%$ [7]. A study performed by Yuan-quan and Jia-jia employed a cluster analysis to explore the impact of urbanization scale distribution on regional innovation efficiency; he found that there is an "inverted U" association between urbanization scale distribution and regional innovation efficiency. In addition, an optimal city size distribution can maximize the efficiency of regional innovation. Inefficient or excessive city size is not conducive to the improvement of regional innovation efficiency [8]. Gao (2015) performed empirical research of more than 287 prefecture-level cities; the larger the scale of the city, the stronger its innovation ability and performance [9]. Guo et al. (2015) described the city size in terms of population density, innovation output, and patent strength; they explored the relationship between population density and regional innovation output using spatial economic methods. The study found that when the population of the city is between 50 and 90 million, the innovation output level of the city is the highest, and there is a significant "inverted U" relationship between the city size and innovation [10]. In the two key factors such as agglomeration effect and crowding effect, many scholars explored that the relationship between population density and regional innovation output is not necessarily driving a linear relationship which depends on the comprehensive outcome of both agglomeration effect and crowding effect. Recent research work by Min and Changquan (2019) addresses that in certain regions when the population density increases excessively, it will lead to the phenomenon of excessive agglomeration and the generation of congestion effect in the region [11]. Additionally, Ye et al. (2016) propagate that there is a threshold effect between the population density and regional innovation efficiency by analyzing the relationship between the population density and regional innovation efficiency [12]. Some scholars discussed big data and smart cities such as analysis of dimensionality reduction techniques on big data and deep learning for future smart cities. Kumar et al. (2021) think that smart cities have become the mainstream of urbanization [13]. Ram et al. (2021) discussed security-by-design (SbD) concepts in the energy harvester technologies for sustainable and secured IoT with uninterrupted energy resource smart villages and smart cities [14]. Liu et al. (2017) discussed enormous data from IoT is stimulating our cities to become smarter than ever before [15].

From the above studies, a wide range of census by numerous scholars noticed that there is an extensive and complicated relationship existed between urban innovation output and urban scale, population density, and the nonlinear relationship between population density and regional innovation output. However, few studies have explored the threshold effect of urban size and population density on regional output. Therefore, based on the panel data of 225 cities at the prefecture level and above in 2007-2016, this study examined the impact of city size and population density on regional innovation output. In contrast, the existing literature on the innovation of this research lies in various ways. Firstly, this study considers an empirical analysis of the cross effect of innovation output on urban size and population density to examine whether there is an alternative or complementary relationship between urbanization size and population density. For this purpose, we add the cross terms of urbanization size and population density into the empirical analysis model to examine whether the effectiveness of one variable is conditional on another variable and if so whether there is a complementary or alternative relationship.

Secondly, this study considers an empirical analysis on whether there is a threshold effect on the regional innovation output of urban scale and population density. Considering that the improvement of urban scale and population density will promote the improvement of urban innovation capability, such improvement may depend on the development of urban scale and population density to a certain extent, which means that there may be a "threshold effect" in the improvement effects of urban scale and population density on innovation competency. Therefore, this study uses city size and population density as threshold variables and utilizes the bootstrap method to sample 500 times, to measure the threshold effect of regional innovation output of city size and population density.

\section{The Internal Mechanism of the Impact of Urban Scale and Population Density on Innovation Output}

Developed cities are the core hub of innovations such as human resources, scientific research, technological advancement, and information resources. The impact of urbanscale expansion on innovation output is primarily reflected in many aspects such as the expansion of urban scale helps to promote the agglomeration of regional innovation output, expertise, and boost innovation patterns. Many researchers believe that the expansion of city scale promotes regional innovation such as human resources, intellectual property protection, product professional test, and intermediate and technical information services. In addition, it has been widely acknowledged that the Chinese first-tier cities attracted millions of highly skilled workers that resultantly consolidate the city innovation output on a larger scale and also promote the technological innovation research to rapidly diminish the innovation cost and time. The latest study by Gerlach (2009) shows that when conducting innovation activities, most enterprises prefer to choose regions with higher concentration, because these enterprises have rich shared resources, compared with the regions with low concentration; these regions can enable enterprises to save innovation costs and reduce their risks [16].

Secondly, the expansion of the urban scale will help to enhance the matching degree and supply of the labor market. Consequently, the innovation subjects can match highly skilled talents and thus promote the development of regional innovation [17]. Another study by Parrotta et al. (2014) shows that the abundance of talent selection will significantly reduce the innovation robustness of enterprises [18]. The 
refinement of the matching degree of the labor market will also help to promote the structural matching and accumulation velocity of knowledge creation and promote innovation output. Thirdly, the expansion of city scale means the expansion of market scale and the continuous expansion of the demand of innovation outcomes. All these factors encourage enterprises to increase the absorption capacity and integration of innovation resources that improve their innovation capabilities.

The impact of urban population density on innovation output is mainly through the following ways: (i) the spatial proximity effect brought by the increase of population density is conducive to innovation output. The spatial proximity effect can create highly skilled talents to better communicate and boost the frequency of communication that is conducive to the spillover and dissemination of knowledge, especially invisible knowledge and noncoding knowledge to promote the generation of innovation. (ii) The social network effect brought by the increase of population density is beneficial to innovation output with all kinds of innovative outcomes obtained in the city. Moreover, establishing a formal contact is becoming a perfect social network, in the long-term stable cooperative relationship. In addition, it helps to reduce the contract cost caused by the uncertainty and inconsistency of information, promote the sharing of information resources, and improve the regional innovation ability. A similar study by Glaeser (1999) shows that even with the development of modern technology, people can communicate in a more diversified and convenient way. However, in social networks, the impact of informal relations on knowledge and information dissemination will not be neglected, because it cannot replace face-to-face communication, which is inevitable in a formal contract [19].

\section{Empirical Study on the Impact of Urban Scale and Population Density on Innovation Output}

3.1. Variable Selection and Data Analyzation. In this paper, we add one explanatory variable that is innovation output (patent density) and describe it as the number of invention patents per 10000 people. Conventionally, the main indicators to measure the innovation output include the number of patent licenses, the number of patent applications, the transfer of patent use fees and royalties, and the technical market turnover. Moreover, it is difficult to measure accurately and effectively the regional innovation output, because the number of patent applications contains a large number of unlicensed patents. In addition, patents include invention licenses, utility model patents, and design patents. The amount of patent license can be considered to estimate the regional innovation output and information more effectively and efficiently. However, although the indicators such as royalty and technical market turnover with other data sources can more fully reflect the regional innovation output.

Explanatory variables include the size of a city that is represented by the resident population of the municipal district.
Innovation is primarily a city phenomenon. The high concentration of various facilities in the city is more conducive to the development of innovation. The larger the city scale, the greater the agglomeration and attraction of innovative highly skilled talents. On the other hand, if the city scale is large, it is relatively easier to acquire relevant professional machinery and equipment. In addition to this, large-scale cities provide access to obtain relevant intellectual support and innovation-related services. At the same time, large city sizes are substantial to accommodate the division of labor and cooperation and various information exchanges related to innovation. Presumably, the city size helps to improve the process efficiency resulting from innovation output.

3.1.1. Population Density-Regional Innovation Output Nexus. This research uses the employment-population density of the municipal district to measure the employment-population of the municipal district, the built-up area of the municipal district. Because there are different categories and segments of the districts (mainly urban districts) and built-up areas in China, the population density is measured based on the urban district's population density and agglomeration due to the inclusion of a large number of rural areas. Substantially, most of the economic activities of a city are mainly concentrated in the urban start-up areas. Therefore, this paper uses the number of the urban population divided by the start-up area to better measure the regional population concentration $[20,21]$. Knowledge exchange and accumulation are the basis of innovation. Moreover, the higher the population density, the more conducive the formation of a compact space distance and intensive social relationship network. Furthermore, it is convenient for people to exchange knowledge, disseminate implicit information and accumulate knowledge, and improve the frequency of contact between people. The higher frequency of contact increases the possibility of knowledge dissemination and innovation, which in turn promotes the enhancement of regional innovation efficiency and regional innovation output.

3.1.2. Research and Development Investment. In this section, we focus on the local financial science and technology expenditure of the municipal government to measure the government's financial support for technological innovation. On the one hand, the financial science and technology-related expenditure of the local government could directly provide financial support and related preferential policies for urban innovation activities, which increases the urban innovation input and thus improves the urban innovation output. On the other hand, the government's investment in innovation can serve as a "good gesture" and policy guidance. It is reflecting the local government's support capacity and image for innovation, which helps to strengthen the confidence of enterprises in investment and lead enterprises to increase more investment in research and development as well as create more innovation output. At the same time, the "leverage effect" brought by the government's innovation investment can leverage more private investment; thus, the urban innovation ability becomes stronger. 
3.1.3. The Proportion of the Third Industry. In this section, we use the proportion of the third industry in GDP of municipal districts to measure the cluster development of the service industry that can provide targeted specialized services for innovation activities. It provides legal and advisory services in the process of the patent application that reduces the transaction costs of innovation activities. Furthermore, the development of financial services in the regional industry can effectively reduce the financial cost of enterprises, improve the financing efficiency, and provide more investment in innovation funds for enterprises. Therefore, the research by Yang and Bao (2019) also found that the specialization and diversification of producer services have a significant role in promoting urban innovation [22].

3.1.4. Foreign Direct Investment. In the amount of foreign direct investment in a municipal district compared with the domestic level, foreign direct investment can bring more advanced technology, highly skilled management, and more proficient innovation development. On the one hand, the proportion of high-quality foreign innovation capital has increased, which can directly improve the regional innovation efficiency with its efficient innovation ability. On the other hand, the foreign direct investment will bring technology spillover to the region and promote the innovation ability and innovation efficiency of the region through demonstration effect, competition effect, human capital flow effect, and correlation effect [23]. Foreign direct investment not only brings a large amount of capital investment but also brings advanced technology and highly skilled talent to boost domestic technological progress. It was in a relevant study where Miao (2009) used panel data to conduct spatial measurement research and found that FDI caused imitation effect and competition effect, which had a significant positive impact on innovation [24].

3.2. Model Specification. Based on the previous theoretical analysis, we constructed the following empirical model:

$$
\text { Patent density }{ }_{i t}=\alpha_{0}+\alpha_{1} \text { size }_{i t}+\alpha_{2} \operatorname{density}_{i t}+\sum \alpha_{k} X_{k i t}+\varepsilon_{i t} \text {, }
$$

where $i=1,2, \cdots, N$ represents the different cities, $t=1,2$, $\cdots, T$ represents the cities, $t$ represents the years, patent density $_{i t}$ is the patent density, i.e., the number of invented patents authorized per 10000 people, and is the explained variable of the model, size ${ }_{i t}$ represents the city size, density ${ }_{i t}$ represents population density, and $X_{k}$ represents a series of control variables.

Likewise, we also investigate whether there is a substitution relationship between urban size and population density in the case of small city size. Nevertheless, the improvement of population density can make up for the low innovation efficiency brought by the small city size. In the case of insufficient population density, the expansion of the urban scale makes up for the problem of low innovation output caused by insufficient communication due to low population density. Therefore, we add the interactive terms of urban size and population density into Equation (1) to obtain the final expression of the theoretical model in this paper:

$$
\begin{aligned}
\text { Patent density } & =\alpha_{0}+\alpha_{1} \text { size }_{i t}+\alpha_{2} \text { density }_{i t}+\alpha_{3} \text { size }_{i t} \\
& * \operatorname{density}_{i t}+\sum \alpha_{k} X_{k i t}+\varepsilon_{i t} .
\end{aligned}
$$

3.3. Data Source and Descriptive Statistics. In this paper, we used unbalanced data due to the lack of relevant observations for some prefecture-level cities. For the administrative region adjustment of relevant cities in the sample range of 20072016, it is necessary to adjust the relevant data to maintain the consistency and accuracy of the data. Therefore, this study conducts essential steps as follows. Firstly, the management area of some cities has changed significantly, which may be due to the adjustment of different zones. Therefore, we have eliminated the sample cities with land area change of more than 5\% during the sample period. Secondly, for some indicators, data of individual cities are missing. To overcome this issue, this study uses the method of moving average to carry out the edge in processing by referring to the processing methods of relevant research. Thirdly, some sample cities with substantial data deficiency, such as Sansha City, Baying City, and Fangchenggang City, were excluded from the study. At the same time, taking into account the time of innovation (assuming 1 year), it generally takes 1 year to apply for and obtain patents. In this paper, the number of invention patents measured by regional innovation output since the regression equation is 2005-2014.

The data used in this paper are obtained from the China Urban Statistical Yearbook, China Regional Economic Statistical Yearbook, and China Statistical Yearbook from 20062016 issued by the National Bureau of Statistics as well as the relevant annual statistical yearbook of the provinces where the relevant cities are located. Due to the lack of some of the missing data supplemented by the statistical yearbook of the city where they are located, some of the data cannot be obtained and supplemented by the linear interpolation method. Taking into account the impact of inflation, the GDP deflator is added to take 2007 as the base period to conduct the deflator processing on R\&D, FDI, and other data. The summary statistics are presented in Table 1.

3.4. Analysis of Empirical Results. This study includes the panel data of 230 each prefecture-level city and over 10 years. We assume that $i>t$; the individuals of the samples in each period are the same, with balanced panel data. Since the number of individuals in this panel is much larger than the time dimension, therefore, we run a regression model without a unit root test. The basic model of panel data regression is as follows:

$$
\text { Patent density }{ }_{i t}=\alpha_{0}+\alpha_{1} \operatorname{size}_{i t}+\alpha_{2} \operatorname{density}_{i t}+\sum \alpha_{k} X_{k i t}+\varepsilon_{i t} .
$$

In the empirical section, this paper employs a variety of panel data regression models, such as mixed-effects model, individual random-effects model, and fixed-effects model. The specific model shall be determined through relevant tests. Firstly, the appropriate estimation method is 
TABLE 1: Summary of descriptive statistics.

\begin{tabular}{|c|c|c|c|c|c|}
\hline Variables & Observations & Mean & Std. & $\operatorname{Max}$ & Min \\
\hline Patent density (piece/10000 persons) & 2300 & 17.08 & 26.74 & 290.34 & 0.01 \\
\hline City size (10000 persons) & 2300 & 125.01 & 104.64 & 2440.82 & 26.3 \\
\hline Population density (person $/ \mathrm{km}^{2}$ ) & 2300 & 13254.5 & 4402.50 & 28021.88 & 2688.73 \\
\hline $\mathrm{R} \& \mathrm{D}$ investment (ten thousand yuan) & 2300 & 28242.89 & 66778.04 & 954447 & 44 \\
\hline Third industry (\%) & 2300 & 43.07 & 11.02 & 78.66 & 8.58 \\
\hline FDI (ten thousand dollars) & 2300 & 78802.76 & 134140.10 & 983567 & 0.38 \\
\hline
\end{tabular}

TABLE 2: Regression results of fixed-effects model, random-effects model, and mixed-effects model.

\begin{tabular}{lcccc}
\hline Variable & $\begin{array}{c}\text { Model 1 } \\
\text { (fixed-effects model) }\end{array}$ & $\begin{array}{c}\text { Model 2 } \\
\text { (random-effects model) }\end{array}$ & $\begin{array}{c}\text { Model 3 } \\
\text { (mixed-effects model) }\end{array}$ & $\begin{array}{c}\text { Model 4 } \\
\text { (add cross product) }\end{array}$ \\
\hline Size & $0.193^{* * *}(7.71)$ & $0.183^{* * *}(4.42)$ & $0.105^{* *}(3.65)$ & $0.164^{* * *}(4.42)$ \\
Density & $0.285^{* * *}(16.78)$ & $0.261(0.90)$ & $0.424^{* * *}(8.95)$ & $0.296^{* * *}(7.10)$ \\
R\&D & $0.235^{* * *}(14.46)$ & $0.250^{* * *}(12.20)$ & $0.271^{* * *}(9.70)$ & $0.246^{* * *}(6.15)$ \\
Third industry & $0.131^{* * *}(4.57)$ & $0.127^{* * *}(3.81)$ & $0.092(2.81)$ & $0.091^{* *}(3.01)$ \\
FDI & $0.042(1.31)$ & $0.045(1.21)$ & $0.062(1.16)$ & $0.116(1.11)$ \\
Size $*$ density & & & $0.011(0.48)$ & $0.121^{* * *}(8.8)$ \\
Constant & $0.035^{* * *}(2.80)$ & $0.2444(6.20)$ & 0.525 & $0.069^{* *}(3.52)$ \\
$R$-squared & 0.374 & 0.394 & 0.331 \\
\hline
\end{tabular}

$t$ value in brackets. $*$ indicates significance under $10 \%$ significance level. $* *$ indicates significance under $5 \%$ significance level, $* * *$ indicates significance under $1 \%$ significance level.

determined effectively: $B-P \quad$ test (chibar2 $(01)=2126.92$, Prob $>$ chibar2 $=0.0000)$ and LR likelihood ratio (LR test of sigma). The results of the $(u=0$ : chibar2 $(01)=868.50$, Prob $\geq$ chibar $2=0.000) \quad$ test indicated that it significantly rejected the original assumption at the level of $1 \%$, indicating that individual random-effects model should be selected between mixed-effects model and individual random effect. Therefore, this paper employs the fixed-effects model with the best estimation effect as the main illustration object. As a comparison, the regression results of the random-effects model and the mixed OLS model are also presented in Table 2. Similarly, we add the cross terms of urban size and population density in model 4 to investigate whether there is a mutual or one-way relationship between urban size and population density.

The results indicate that urban scale has a significant and positive effect on innovation output at the level of $1 \%$. It shows that the larger the city scale, the more innovative output factors such as people, enterprises, and industries accumulation. On the other hand, the urban scale provides a more suitable environment for innovation, which is conducive to the development of the latest science and advanced technology. At the same time, the larger the scale of the city, the better for people to learn knowledge, develop their skills, promote the formation of human capital, and ultimately promote the innovation ability of the city. The increase of the city scale is beneficial to the resembling and curtailing of the knowledge of different innovative groups, improving the efficiency and quality of knowledge cognition among different groups which helps in reducing the cost of science and technology. Likewise, innovation activities need to consoli- date equipment and information to find people for division of work cooperation and information exchanges. The application and transaction of patents need the support of external service institutions. In large-scale cities with a large number of employees and a developed service industry, these demands are easier to meet and obtain high-quality services and support.

On the other hand, the results from the proposed model illustrate that population density has a significant and positive effect on regional innovation output at the level of $1 \%$. The research and development (R\&D) personnel in densely populated cities have relatively more person-in-person communication opportunities, which is beneficial to the development of innovative ideas. The higher the population density, the shorter the interaction distance and time, while more opportunities and lower costs for the communication between people in different industries and disciplines. In addition, it is easier for individuals with different knowledge to exchange ideas by searching for partners to improve the efficiency of knowledge exchange and promote the creation of knowledge. The increase in urban population density also contributes to the rapid expansion and exchange of innovative ideas and outputs. Geographical distance plays a distinct role in the process of knowledge exchange and innovation cooperation. The distance between people will affect the intensity of knowledge exchange, innovative cooperation behavior, and propagation of implicit knowledge. However, the theoretical part also supports that the spillover effect and proximity effect affect the probability and quality of innovation cooperation and information exchange. Moreover, the study finds that if the population density is higher, 
TABLE 3: Results of the robustness test.

\begin{tabular}{lccccc}
\hline Variable & Model 1 & Eastern & Model 2 & Middle part & Western \\
\hline Size & $0.121^{* * *}(3.21)$ & $0.281^{* * *}(2.29)$ & $0.213^{* * *}(2.87)$ & $0.121^{* * *}(2.67)$ & $0.298^{* * *}(3.12)$ \\
Density & $0.241^{* * *}(6.71)$ & $0.412^{* * *}(8.22)$ & $0.367^{* * *}(9.13)$ & $0.712^{* * *}(10.21)$ & $0.319^{* * *}(8.32)$ \\
R\&D & $0.235^{* * *}(14.46)$ & $0.312^{* * *}(21.23)$ & $0.209(1.54)$ & $0.281(1.02)$ & $0.235^{* * *}(14.46)$ \\
Third industry & $0.131^{* * *}(4.57)$ & $0.131^{* * *}(4.57)$ & $0.131^{* * *}(4.57)$ & $0.131^{* * *}(4.57)$ & $0.131^{* * *}(4.57)$ \\
FDI & $0.043(1.09)$ & $0.381(0.32)$ & $0.124(1.01)$ & $0.023^{* *}(1.96)$ & $0.121(0.82)$ \\
Constant & $0.035^{* * *}(2.80)$ & $0.244^{* * *}(6.20)$ & $0.011(0.48)$ & $0.069^{* *}(3.52)$ & $0.050^{* * *}(4.35)$ \\
$R$-squared & 0.374 & 0.394 & 0.525 & 0.331 & 0.479 \\
\hline
\end{tabular}

$t$ value in brackets. $*$ indicates significance under $10 \%$ significance level. $* *$ indicates significance under $5 \%$ significance level. $* * *$ indicates significance under $1 \%$ significance level.

it will ultimately be favorable to promoting social proximity, enriching the knowledge exchange channels between innovation outputs, and improving the multidimensional exchange and interaction between innovation contents [25]. Likewise, the urban population density promotes the spillover of innovation knowledge among subjects for the purpose to improve the level of regional innovation. The higher the population density, the more easily the agglomeration economic externality will be obtained. The implicit knowledge related to innovation is often before some core employees, and the regions with high population density can effectively promote the flow and exchange of people and accelerate the spread of tacit knowledge.

Finally, the result of the cross product of urban size and population density is significant and positive, indicating that the impact of urban size and population density on innovation output is interdependent and contribution is interactive. There is a significant positive association between the two variables, one of which is essential to the other. It shows that the impact of urban population density cannot be ignored when considering the promotion of urban scale on innovation output. If the city size is too small and has a high population density, it is difficult to play a role in promoting the increase of innovation output. On the other hand, if the size of the city is large, but there is no certain population density support, it is difficult to form a sufficient agglomeration effect to promote the increase of regional innovation output.

3.5. Robustness Test. To further investigate the robustness of the results and ensure the rationality of the model accuracy and reliability, this study utilizes the following regression results to crosscheck the coefficients of all the selected variables. The robust estimates were performed for the following regression:

Firstly, we replace the main explanatory variable city size of this study. In the previous study, the resident population of municipal districts was used to measure the city size. Next, this study uses the total GDP of urban districts as the proxy variable to measure the city size and estimate the relevant measurement test results (model 1). Secondly, previous studies at the national level have shown that urban size and population density have a significant positive impact on technological innovation.
Thirdly, we exclude four municipalities from the model; the remaining 226 cities above prefecture level were brought into the panel data for estimation (model 3). From the estimation results, the robustness of the model is illustrated in Table 3. The results illustrate that the positive impact of urban size and population density on technological innovation still exists and is consistent with previous analysis. The results of most explanatory variables are robust and accurate in the fixed-effects panel model.

The main difference is that in model 2, the estimation results of subsamples in the eastern, central, and western regions are different to some extent. The impact of regional research and development (R\&D) investment intensity on regional innovation efficiency is different between eastern, central, and western regions. From the sample regression results, the impact of regional $R \& D$ input intensity on regional innovation output is not significant in the subsample estimation results of the central region and western region, while the result of the eastern region is significantly positive. In this case, the input efficiency of innovation resources in the central and western regions is tropical except for the eastern region, while the intensity of $\mathrm{R} \& \mathrm{D}$ input has not been effectively transformed into actual innovation output. Since there are many historical debts of innovation investment in the central and western regions, hence, it is difficult to induce benefits from $R \& D$ investment. It is possible that the intensity of R\&D investment has not yet reached a certain threshold and cannot play an effective role.

In this section of the paper, the result reveals that foreign direct investment (FDI) has a significant impact on the innovation output of cities in the western region at the level of 5\%, but has no significant impact on cities in the eastern region and the central region, respectively. This may be the eastern and central regions are rich in urban innovation resources and have a relatively high level of innovation capacity and efficiency, resulting in an insignificant spillover effect of foreign direct investment on regional innovation output. Furthermore, for the cities in Western China, their innovation resources are relatively scarce, and their innovation ability and efficiency are relatively low. On the one hand, the inflow of FDI increases the regional innovation input; on the other hand, the technology spillover effect and competition effect enhance the regional innovation output. 
TABLE 4: Threshold effect self-sampling inspection.

\begin{tabular}{lccccccc}
\hline & Variable & $F$ & $P$ & BS & $1 \%$ & $5 \%$ & $10 \%$ \\
\hline \multirow{2}{*}{ Size } & Single threshold test & $21.038^{* * *}$ & 0.033 & 300 & 20.479 & 18.500 & 18.292 \\
& Double threshold test & 7.023 & 0.197 & 300 & 11.276 & 10.574 & 8.842 \\
\multirow{3}{*}{ Density } & Single threshold test & $34.622_{* * *}$ & 0.007 & 300 & 14.684 & 11.965 & 8.442 \\
& Double threshold test & $22.498^{* *}$ & 0.023 & 300 & 15.269 & 8.201 & 6.066 \\
& Triple threshold test & 4.348 & 0.211 & 300 & 10.034 & 6.412 \\
\hline
\end{tabular}

\section{Research on the Threshold Effect of Urban Scale and Population Density on Innovation Output}

Although the enhancement of urban scale and population density will promote the improvement of urban innovation ability, such improvement may depend on the development of urban scale and population density to a certain extent, i.e., the development effect of urban scale and population density on innovation ability may have a "threshold effect." Therefore, this paper takes city size and population density as threshold variables and uses the bootstrap method to sample 500 times to test the threshold effect of explanatory variables.

4.1. Threshold Effect Test. First, we need to determine whether the threshold effect exists, and select the appropriate threshold number as well as the threshold value. Based on $F$ statistics and 300 "self-sampling," the $P$ values reported in Table 4 indicate that when the city size is used as a threshold variable, the $F$ statistics value (21.038) of the single threshold test is greater than the $1 \%$ horizontal threshold (20.479). However, the $F$ statistics value of the double threshold test (7.023) is less than the $10 \%$ significance level threshold value (8.842). Therefore, it can be considered that there is only one threshold effect in size.

In addition, the study determines the estimate of the threshold and the 95\% confidence interval. See Table 5 for further details. The single threshold estimate for city size is 293.430 , and the $95 \%$ confidence interval is [39.000, 326.400]. The first threshold of population density is 17197.297, and the second threshold is 13136.986. The likelihood ratio function is illustrated in Figure 1 that shows the estimates and confidence intervals of the threshold.

4.2. Threshold Model. Equation (1) can only reflect the comprehensive static effect of urban scale on regional innovation output; the threshold panel model is further explored to analyze the dynamic effect of urban scale and population density variations on regional innovation output. According to the relevant literature, the two-stage least squares method proposed by Hansen (1999) is used to estimate the data model of the threshold panel. The setting equation of the single threshold regression model is as follows:

Patent density $_{i t}=\alpha_{0}+\alpha_{1}$ size $_{i t}+\alpha_{2}$ density $_{i t}+\sum \alpha_{k} X_{k i t}+\varepsilon_{i t}$.
TABLE 5: Threshold estimation results and confidence interval.

\begin{tabular}{lccc}
\hline Variable & $\begin{array}{c}\text { The threshold } \\
\text { value }\end{array}$ & $\begin{array}{c}\text { Estimated } \\
\text { value }\end{array}$ & $\begin{array}{c}95 \% \text { confidence } \\
\text { interval }\end{array}$ \\
\hline Size & Ito1 & 293.430 & {$[39.000,326.400]$} \\
& Ito1 & 17197.297 & {$[13892.320$,} \\
Density & & & $18115.310]$ \\
& Ito2 & 13136.986 & {$[86432.016$,} \\
& & $14341.831]$ \\
\hline
\end{tabular}

where $1(0)$ is an indicator function and the value is 1 when the corresponding condition is raised; otherwise, it is 0 .

In contrast, in the actual observation value with the threshold value, the sample observation value can be divided into two intervals: the coefficient of the corresponding range by looking at the marginal coefficients of different intervals. We examine the impact direction and extent of changes in urban size and population density on regional innovation output if there are multiple thresholds; we expand the above equation based on a single threshold.

4.3. Panel Threshold Analysis. Based on the threshold regression analysis, the urban innovation output is considered as the explanatory variable, while the urban scale and population density were added as the threshold variable. On the other hand, research and development (R\&D) investment, the proportion of the tertiary industry, and international direct investment add as the core explanatory variables for threshold regression. The specific inspection results are illustrated in Tables 6 and 7.

Table 6 shows that there are significant innovationpromoting effects on urban-scale expansion. When the urban scale exceeds the threshold value of 293.430, the innovation promotion effect of urban-scale expansion increases significantly, and its effect coefficient increases from 0.128 to 0.233 , indicating that although the expansion of urban scale is favorable to improving urban innovation output. However, for cities of different scales, when the city size is greater than the specific threshold ( 293.430 people $\left./ \mathrm{km}^{2}\right)$, it will further release the innovation promotion effect brought by the expansion of the city scale. In theory, if the city size exceeds a certain threshold, the city will have complete information, communication, and other infrastructures. Moreover, the agglomeration effect of innovative resources, the accumulation effect of human capital, the formation effect of information exchange network, and the improvement effect of transaction efficiency can be effectively exerted. The larger the scale of the city, the easier it is to become the 

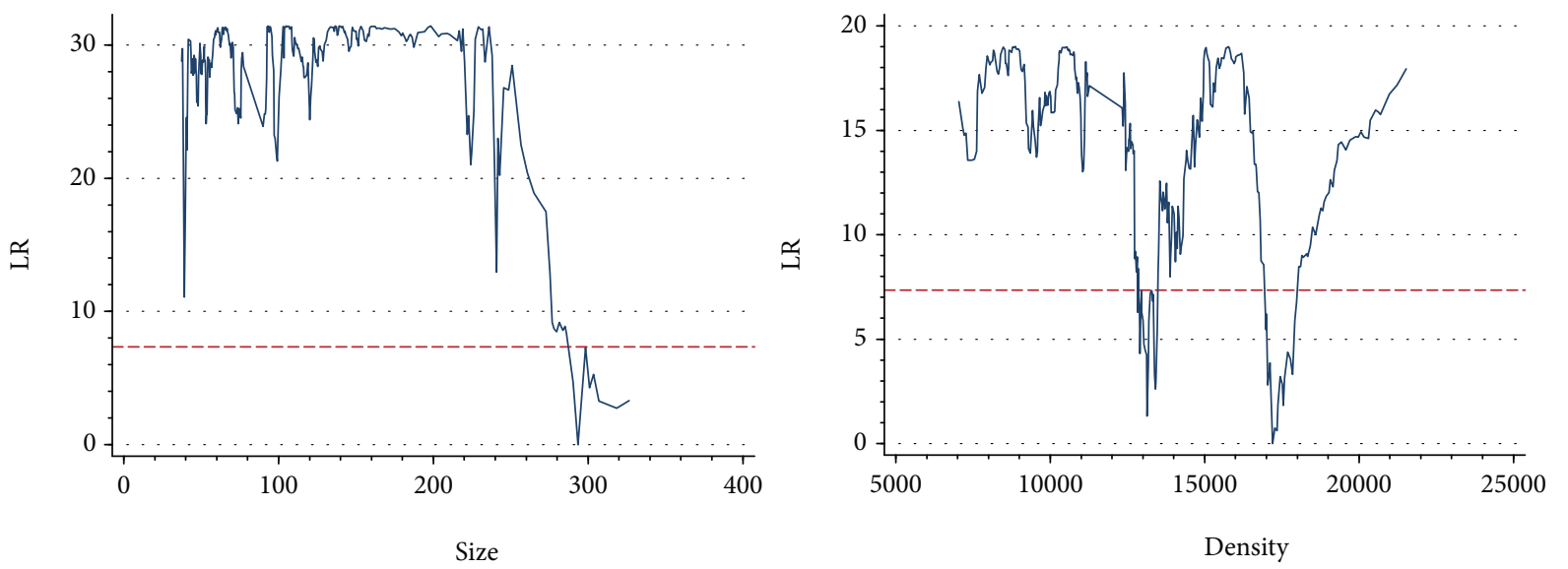

FIGURE 1: Threshold and confidence interval.

TABLE 6: Results of model estimation based on the urban size as a threshold.

\begin{tabular}{lccc}
\hline Variable & Coefficient & Standard error & $t$ \\
\hline Size $\leq 293.430$ & $0.128^{* * *}$ & 0.040 & 3.21 \\
Size $>293.430$ & $0.233^{* * *}$ & 0.059 & 3.98 \\
Density & $0.347^{* * *}$ & 0.123 & 2.81 \\
R\&D & $1.287^{* * *}$ & 0.247 & 5.21 \\
Third industry & $3.218^{* *}$ & 1.634 & 1.97 \\
FDI & 3.092 & 3.031 & 1.02 \\
Constant & $0.218^{* * *}$ & 0.068 & 3.21 \\
$R$-squared & & 0.372 & \\
\hline
\end{tabular}

$t$ value in brackets. $*$ indicates significance under $10 \%$ significance level. $* *$ indicates significance under $5 \%$ significance level. $* * *$ indicates significance under $1 \%$ significance level.

TABLE 7: Model estimation results with population density as the threshold.

\begin{tabular}{lccc}
\hline Variable & Result & $\begin{array}{c}\text { Standard } \\
\text { error }\end{array}$ & $t$ \\
\hline Density $\leq 13136.986$ & $0.216^{* * *}$ & 0.049 & 4.37 \\
$13136.986<$ density $\leq 17197.297$ & $0.431^{* * *}$ & 0.110 & 3.92 \\
Density > 17197.297 & 0.033 & 0.046 & 0.72 \\
Size & $0.347^{* * *}$ & 0.025 & 13.64 \\
R\&D & $0.821^{* * *}$ & 0.250 & 3.29 \\
Third industry & $1.921^{* * *}$ & 0.919 & 2.091 \\
FDI & 0.214 & 0.177 & 1.21 \\
Constant & $0.306^{* * *}$ & 0.062 & 4.91 \\
$R$-squared & & 0.461 & \\
\hline
\end{tabular}

$t$ value in brackets. $*$ indicates significance under $10 \%$ significance level. $* *$ indicates significance under $5 \%$ significance level. $* * *$ indicates significance under $1 \%$ significance level.

concentration of innovation and invention as well as city size will affect the regional human capital accumulation [26]. On the other side, when the city scale is larger, then more various innovative resources will be gathered; also, more dynamic the innovation development as well as the innovation segments will boost likely to produce high-quality innovation results. The empirical study of Miao (2009) also shows that regional innovation activities have an externality [27]. The externality of innovation activities has a certain space scope that innovation activities have agglomeration. The expansion of urban scale is conducive to the agglomeration of innovation elements and the promotion of regional innovation output. The diversity brought by the expansion of urban scales, such as diversity of economic activities, diversity of innovation activities, and diversity of innovation groups, also helps to trigger innovation ideas and improve urban innovation output.

We refer to the population-density cities the first threshold $\left(13136.986\right.$ people $\left./ \mathrm{km}^{2}\right)$ as low-density cities, the population-density cities between the first threshold and the second threshold as medium-density cities (13136.986 people $/ \mathrm{km}^{2}-17197.297$ people $\left./ \mathrm{km}^{2}\right)$, and the populationdensity cities above the second threshold (17197.297 people $\left./ \mathrm{km}^{2}\right)$ as high-density cities. From the regression results in Table 7, we explore that for low-density cities and medium-density cities, the increase of urban population density has a significant positive impact on urban innovation ability. The increase in urban population density can significantly improve the technology of new knowledge in the society, further improve the level of specialization and division of labor [28], and is conducive to improving urban innovation.

However, in high-density cities, increasing population density does not have a significant impact on innovation output. This also indirectly proves that the density of employment agglomeration in some cities in China is too high, and there has been a significant agglomeration diseconomy [29]. This may be due to the lack of economic activities of congestion in high-density cities, such as the increase in commuting costs caused by the congestion effect, resulting in a large number of losses of effective labor commuting and damaging the city's innovation ability. From the perspective of coefficient, the innovation promotion effect of mediumsized cities is significantly greater than that of low-density cities with coefficients of 0.431 and 0.216 , respectively. As 
previously analyzed, the increase in urban population density can promote the sharing of public facilities, facilitate the matching between workers and positions, and promote the speed and scope of the dissemination and exchange of "hidden" knowledge related to innovation. Additionally, the urban city population facilitates the mutual exchange and imitation among organizations and talents. However, the larger population density will highlight crowding and other negative effects as well as make the marginal agglomeration effect caused by the increase of urban population density offset by the marginal crowding effect, which is not beneficial to the improvement of urban innovation output capacity. Therefore, for high-density cities, we should optimize the layout of industry and population within the city to develop multicenter cities. By decentralizing employment in peripheral subcenters, the scale of the urban population is increased, while the commuting distance is greatly reduced, the commuting cost is saved, and the negative impact of the increase in population density is reduced.

\section{Conclusions and Policy Recommendations}

The empirical research shows that the urban size and population density have a significant positive impact on regional innovation output. The impact of urban size and population density on innovation output shows a significant and positive relationship. The empirical results of the threshold panel model show that when the city size exceeds the threshold value of 293.430 million people, the regional innovation output development effect of urban-scale expansion increases significantly. There is a double threshold effect on population density that has a significant positive impact on urban innovation development with the increase of urban population density in low- and medium-sized cities. However, the result also reveals that for high-density cities, the increase in population density will not have a significant impact on innovation output.

Based on the empirical analysis findings, to further enhance the improvement of regional innovation level, this paper proposes the following countermeasures and suggestions: First, our findings suggest that local and central governments should continue to expand the scale of cities, especially small- and medium-sized cities. We follow the laws of urbanization development, urban-scale expansion, and industrial development that actively promote population urbanization. Specifically, starting from the solution of housing security, children's education, medical facilities, pension, and other issues, we should formulate relevant support and support policies to promote the "new citizens" to stay in the city. Particularly, in the context of rising housing prices, it is necessary to further improve the "government-led, market participation" housing system, increase the supply of affordable housing, and improve the quality of affordable housing. In addition, small- and medium-sized cities should cultivate pillar industries according to local conditions, gather population through industrial development, and improve the ability to create employment and acquire labor force. Secondly, while increasing the size of the city, the policymakers actively increase the population density of the city promote the expe- dition of population urbanization, consciously improve the population density of the city, avoid the large-scale inefficient outward spread of the city, improve the population and economic activities, and boost the utilization efficiency of land resources. Thirdly, smart growth theory and dense city concept should be merged into urban planning and development concept. To elude the crowding effect brought by megacities, the expansion of start-up area or restriction of population inflow currently adopted at the cost of damaging the regional innovation ability. It is necessary to integrate smart growth theory and dense city concept into urban planning and development concept into all aspects of urban development and management, to reduce the crowding effect while increasing urban population density.

\section{Data Availability}

The data used to support the findings of this study are included within the article.

\section{Conflicts of Interest}

The authors declare that they have no competing interest.

\section{Acknowledgments}

This work was financially supported by the research projects of the social science and humanity on Young Fund of the Ministry of Education of China (18YJCZH163) and the Philosophy Planning Project of Anhui Province (AHSKZ2019D028).

\section{References}

[1] Z. Rui, J. Limin, G. Xu, X. Zhibang, and D. Ting, "The relationship between urban space growth and population density," Journal of Geography, vol. 75, no. 4, pp. 695-707, 2020.

[2] A. Pred, "Interfusions: consumption, identity and the practices and power relations of everyday life," Environment and Planning A: Economy and Space, vol. 28, no. 1, pp. 11-24, 1996.

[3] B. W. Duncan, S. Boyle, D. R. Breininger, and P. A. Schmalzer, "Coupling past management practice and historic landscape change on John F. Kennedy Space Center, Florida," Landscape Ecology, vol. 14, no. 3, pp. 291-309, 1999.

[4] G. Marella, V. Antoniucci, and C. D'Alpaos, "How regulation affects innovation: the smart grid case at urban scale," in 22nd Annual European Real Estate Society Conference, Istanbul, Turkey, 2015.

[5] A. Wojcik-Popek, "Green innovation in urban scale: activation of small cities through horticultural exhibitions in Berlin/Brandenburg Metropolitan Region," IOP Conference Series: Materials Science and Engineering, vol. 471, p. 112100, 2019.

[6] F. G. van Oort and N. S. Bosma, "Agglomeration economies, inventors and entrepreneurs as engines of European regional productivity," Annals of Regional Science, vol. 51, no. 1, pp. 213-244, 2012.

[7] G. A. Carlino, S. Chatterjee, and R. M. Hunt, "Urban density and the rate of invention," Journal of Urban Economics, vol. 61, no. 3, p. 419, 2007. 
[8] L. U. Yuan-quan and Q. Jia-jia, "Study on the influence of urban scale distribution of China on regional innovation efficiency," Economic Survey, vol. 35, no. 6, pp. 1-7, 2018.

[9] X. Gao, "Urban scale, human capital and urban innovation capacity in China," Social Sciences, vol. 3, pp. 49-58, 2015.

[10] J. Guo, H. Ning, and T. Shen, "Employment density and innovation-based on the spatial measurement of prefecture level cities in China," Economic and Management Research, vol. 36, no. 11, pp. 40-46, 2015.

[11] D. Min and L. Changquan, "Agglomeration effect, population mobility and urban growth," Population and Economy, vol. 21, no. 6, pp. 44-56, 2019.

[12] X. Ye, T. Changqi, and D. Hui, "Empirical study on the coupling of regional industrial innovation and industrial upgrading - taking the Pearl River Delta region as an example," Scientific Research Management, vol. 36, no. 4, pp. 111-119, 2016.

[13] P. Kumar, R. Kumar, G. Srivastava et al., "PPSF: a privacypreserving and secure framework using blockchain-based machine-learning for IoT-driven smart cities," IEEE Transactions on Network Science and Engineering, p. 1, 2021.

[14] S. K. Ram, B. B. Das, K. Mahapatra, S. P. Mohanty, and U. Choppali, "Energy perspectives in IoT driven smart villages and smart cities," IEEE Consumer Electronics Magazine, vol. 10, no. 3, pp. 19-28, 2021.

[15] Y. Liu, X. Weng, J. Wan, X. Yue, H. Song, and A. V. Vasilakos, "Exploring data validity in transportation systems for smart cities," IEEE Communications Magazine, vol. 55, no. 5, pp. 26-33, 2017.

[16] M. Gerlach, "Controlling in einem deutsch-chinesischen Joint Venture - Ein Erfahrungsbericht," Controlling \& Management, vol. 53, no. 2, pp. 94-98, 2009.

[17] S. Yu, "Heterogeneous labor, matching effect and industrial agglomeration," Economic and Management Review, vol. 4, pp. 44-51, 2016.

[18] P. Parrotta, D. Pozzoli, and M. Pytlikova, "Labor diversity and firm productivity," European Economic Review, vol. 66, no. C, pp. 144-179, 2014.

[19] E. L. Glaeser, "Learning in cities," Journal of Urban Economics, vol. 46, no. 2, pp. 254-277, 1999.

[20] Q. Guo and H. Canfei, "Density, distance, segmentation and urban labor productivity: an empirical study based on China's 2004-2009 urban panel data," China Soft Science, no. 11, pp. 82-91, 2014.

[21] Z. Haoran and Y. Zhong, "Infrastructure, spatial spillover and regional TFP: an empirical study based on the Dupin model of 266 cities in China," Economist, vol. 2, no. 2, pp. 61-67, 2018.

[22] R. Yang and J. Bao, "Can agglomeration of productive services effectively promote urban innovation," Discussion on Modern Economy, no. 4, pp. 80-87, 2019.

[23] L. Zheng, S. Yang, and H. Bin, "Does FDI inhibit or improve the efficiency of regional innovation in China?- analysis based on the inter provincial spatial panel model," Economic Management, vol. 39, no. 4, pp. 8-21, 2017.

[24] T. Song, "Empirical Analysis on Regional Industry Brand Effect,Externality and Industrial Agglomeration-Based on Spatial Model of China's Provinces," Economic Management Journal, vol. 37, no. 8, pp. 35-44, 2015.

[25] L. Guoqing, Z. Gang, and G. Nana, "Dynamic evolution analysis of innovation network based on geographical proximity and social proximity - taking China's equipment manufactur- ing industry as an example," China Soft Science, vol. 5, pp. 97106, 2014.

[26] C. Kaiming, Z. Yafei, and C. Long, "Effect decomposition and mechanism analysis of China's urbanization on energy consumption," Geographic Science, vol. 36, no. 11, pp. 1661$1669,2016$.

[27] F. Miao, Spatial Measurement and Threshold Regression Analysis of Technology Spillover, Huazhong University of Science and Technology, 2009.

[28] K. Shanzi and Y. Delong, "Causal relationship and determinants of industrial agglomeration and urban labor productivity: an analysis of the spatial econometric simultaneous equation of Chinese cities," Research on Quantitative Economy, Technology and Economy, vol. 25, no. 12, pp. 3-14, 2008.

[29] S. Hongjian and W. Houkai, "Density effect, optimal urban population density and intensive urbanization," China Industrial Economy, vol. 10, pp. 5-17, 2013. 